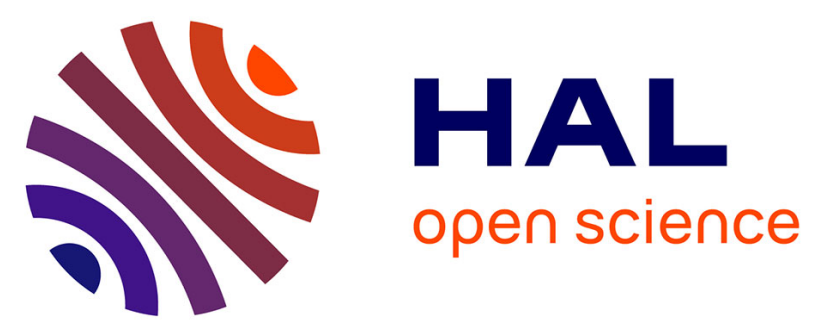

\title{
Texture Rendering Strategies with a High Fidelity - Capacitive Visual-Haptic Friction Control Device
}

Eric Vezzoli, Thomas Sednaoui, Michel Amberg, Frédéric Giraud, Betty Lemaire-Semail

\section{- To cite this version:}

Eric Vezzoli, Thomas Sednaoui, Michel Amberg, Frédéric Giraud, Betty Lemaire-Semail. Texture Rendering Strategies with a High Fidelity - Capacitive Visual-Haptic Friction Control Device. Haptics: Perception, Devices, Control, and Applications, Jul 2016, London, United Kingdom. 10.1007/978-3319-42321-0_23. hal-01341981

\section{HAL Id: hal-01341981 \\ https://inria.hal.science/hal-01341981}

Submitted on 8 Jul 2016

HAL is a multi-disciplinary open access archive for the deposit and dissemination of scientific research documents, whether they are published or not. The documents may come from teaching and research institutions in France or abroad, or from public or private research centers.
L'archive ouverte pluridisciplinaire HAL, est destinée au dépôt et à la diffusion de documents scientifiques de niveau recherche, publiés ou non, émanant des établissements d'enseignement et de recherche français ou étrangers, des laboratoires publics ou privés. 


\title{
Texture Rendering Strategies with a High Fidelity Capacitive Visual-Haptic Friction Control Device
}

\author{
Eric Vezzoli ${ }^{1}$, Thomas Sednaoui ${ }^{1,2}$, Michel Amberg ${ }^{1}$, Frédéric Giraud ${ }^{1}$ and Betty Lemaire-Semail ${ }^{1}$ \\ ${ }^{1}$ Univ. Lille, Centrale Lille, Arts et Metiers ParisTech, HEI, EA 2697 - L2EP - \\ Laboratoire d'Electrotechnique et d'Electronique de Puissance, F-59000 Lille, France \\ ${ }^{2}$ STMicroelectronics, Crolles F38920, France \\ *eric.vezzoli@ed.univ-lillel.fr; \\ thomas.sednaouidst.com; \\ Michel.Ambergeuniv-lillel.fr; \\ \{frederic.giraud, betty.semail\}@polytech-lille.fr;
}

\begin{abstract}
Ultrasonic vibrations of a plate can modify the perception of the friction between a surface and a sliding finger. This principle, coupled with modern position sensing techniques, is able to reproduce textured materials. In this paper, an open loop control through model inversion of the friction force between the finger and the plate is presented. The device incorporating the control system is described, and two different reproduction strategies are formalized to address the reproduction of objects and textures. In the end, a psychophysical experiment evaluating the two control strategies is described.
\end{abstract}

Keywords: ultrasonic, tactile device, ultrasonic vibrations, friction control

\section{Introduction}

In recent years, a lot of interest raised around the implementation of haptic stimulation and simulation. Many technologies are available to provide stimulation on a finger touching or sliding on a surface. However, few of those shown a promising opportunity for a coupling with touchscreen and the consequent aim for integration in the mobile world. Friction modulation techniques, namely electrovibration [1], [2], electroadhesion [3] and ultrasonic vibrations [4] are among this group. Their principle relies on the modulation of the friction between a finger sliding on an active surface. Electrovibration and electroadhesion exploit the electrostatic attraction generated between the finger and the plate, the first through the application of a voltage and the second through the application of a current. Both of them enhance the friction between the finger and the plate. On the other hand, ultrasonic vibrations of a plate reduce the friction between the finger and the surface. The reduction of friction was firstly explained through the squeeze film effect [4], later, a more mechanical explanation of the phenomenon was introduced [5], but a reliable modelling is yet to be developed. With the availability of finger detection techniques, the possibility to actively change the friction as a function of the finger position led to the ability to simulate textures [6]. This study focused on the reproduction of a squared grating, but it is lacking a broader approach on object reproduction. As introduced in [7], force plays a fundamental role 
on the reproduction of shapes, and a similar approach on texture reproduction has not been introduced yet.

In this paper, the development and the control of a device able to simulate textures thanks to the friction coefficient modulation between the finger and the vibrating plate is described.

The paper is organized as follows: initially the characteristics of the friction reduction provided by ultrasonic devices are recalled, following, an analysis of previously developed strategies for friction coefficient control is performed. The structure and the open loop control scheme of the device are then described followed by the formalization of the two haptic signal rendering schemes. In the end, a psychophysical experiment exploring the advantages of the two rendering techniques is proposed and the results discussed.

\section{Ultrasonic Lubrication}

The first explanation for the friction reduction in ultrasonic devices was found in the squeeze film effect [8]. The effect relied on the generation of an ovepressured film of gas between the ultrasonic vibrating plate in the range of micrometres and the interface of the finger. The underlying physical phenomenon was firstly modelled by Biet et al [4]. The presence of the squeeze film effect in these devices was lately questioned by Sednaoui et al [9]. The extended study on the characteristics of the friction reduction, reported in [9], identified an empirical model to describe the evolution of the friction modulation. The friction coefficient reduced by the ultrasonic vibration is unable to reach the zero value, and it is reduced with an exponential decay in function of the vibration amplitude. The friction coefficient reaches an asymptotic value for high vibration amplitude where $90 \%$ of the maximum reduction is reached for an amplitude around 2 micrometers, figure 1. It is possible to define the relative friction coefficient $\mu^{\prime}$ as:

$$
\mu^{\prime}(w)=\frac{\mu(w)}{\mu(0)}
$$

Where $\mu(w)$ is the friction coefficient for a given vibration amplitude $w$. In the reported study, $\mu^{\prime}$ was identified as the invariant for similar conditions between different subjects, figure $1 \mathrm{~b}$. The
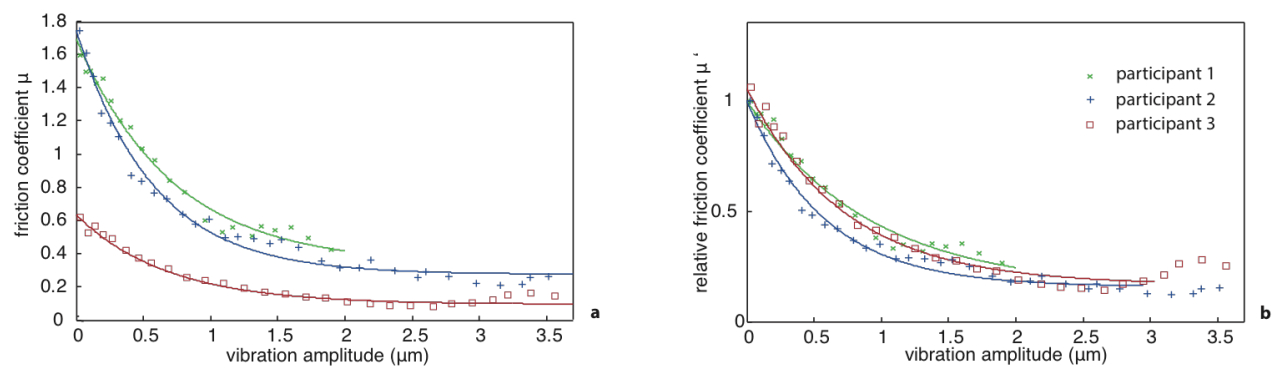

heig ht of the asymp tote of the friction

Fig. 1. Experimental results measured for the friction reduction in ultrasonic device. On the left, the friction coefficient measured through the tribological assessment. On the right, the relative friction coefficient of the same measures.

re-

duction is line- 
arly dependent on the normal force applied. The reduction of relative friction coefficient, $\Delta \mu$, in function of the vibration amplitude can be expressed as:

$$
\begin{gathered}
\Delta \mu(w)=\left(1-\Delta \mu_{1}\right) e^{-b w}+\Delta \mu_{1} \\
\text { With } \Delta \mu_{1}=1.13 F_{n}
\end{gathered}
$$

Where $b$ is an empirical constant with $1.67 \mu \mathrm{m}^{-1}$ as value, $F_{n}$ is the normal force applied by the finger.

\section{Friction Coefficient Control}

In the present section, an analysis of previous implementation of friction coefficient control in ultrasonic devices is presented.

The friction felt by the finger while exploring different surfaces is greatly variable for different subjects: many different parameters influence this force, like the nature of the surface, lipid and water content or the mechanics properties of the finger. Moreover, the friction coefficient may dramatically change within seconds after the contact due to generated occlusion and non-coulomb friction [10]. A closed loop approach of the friction coefficient control was previously developed with the SMARTTAC [11]. This device incorporated a broad bandwidth tribometer around an ultrasonic tactile plate. The lateral and normal force sensors implemented allowed the real time recording and processing of the normal and friction force permitting the implementation of a closed-loop control of the friction coefficient. The system works smoothly for a step of friction coefficient and it is able to maintain the control in the steady state. In this device, the participants' friction coefficient is recorded along the first stroke over the plate and then controlled based on the previous measure.

One issue with this architecture is that a relevant change of the friction due to the generated occlusion or deposited moisture could lead to the saturation of the control. Moreover, the bandwidth of the device is intrinsically limited by the mechanical properties of the fingertip. To effectively control the friction coefficient, the lateral force sensor needs to be able to measure the friction force. Based on the measurements performed in the cited work on different participants, an average rise time of the lateral force of $3.84 \mathrm{~ms}$ was recorded. This value is reflected in a bandwidth of around $90 \mathrm{~Hz}$ for the signal reproduction. The reported value matches the mechanical properties of the fingertip highlighted in [12] and it is in accord with similar measurements performed in [13] and validated for both electrovibration and ultrasonic vibrations in the cited work. The elastic response of the fingertip imposes a bandwidth limit to the closed loop friction coefficient control far below the perceptual bandwidth of the finger. Due to the intrinsic limitation of a direct measurement and control of the lateral force, an open-loop approach will be described in the following sections.

\section{$4 \quad$ High Fidelity Texture Rendering}

In this section, the developed device is described and the strategies of texture reproduction are introduced. 


\subsection{Device}
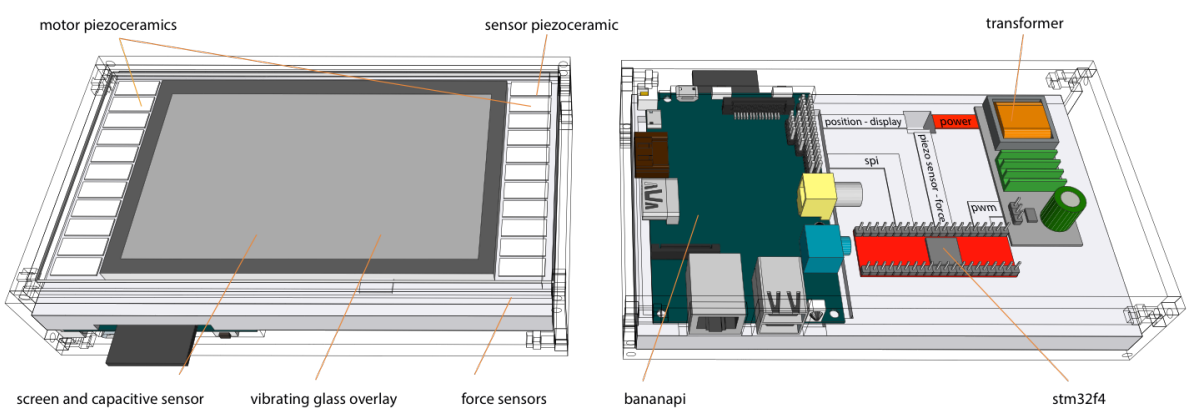

$\mathrm{T}$

he

de-

vice

is

built

arou

nd

the

ba-

nana

pi

Fig. 2. Device structure.

(She

nzhe

$\mathrm{n}$

LeMaker Technology Co. Ltd, China) single board computer featuring a $1 \mathrm{GHz}$ ARM Cortex-A7 dual-core CPU with $1 \mathrm{~GB}$ of ram working in parallel with a microcontroller (stm32f4, STMicroelectronics, France). The single board computer is connected to a 5 inches flat capacitive touch screen (Banana-LCD-5"-TS, Marel, China) providing the finger position input and display output, where the sampling frequency of the finger position is $50 \mathrm{~Hz}$. The communication between the microcontroller and the single board pc is provided by an SPI bus working at $10 \mathrm{kframes} / \mathrm{second}$. 4 flat resistive force sensors (CP 150, IEE, Luxemburg) are placed under the corners of the display and provide the normal force value to the microcontroller. The microcontroller synthesizes a PWM signals to pilot a voltage inverter the motor piezoceramics. In figure 2, the structure of the device is represented.
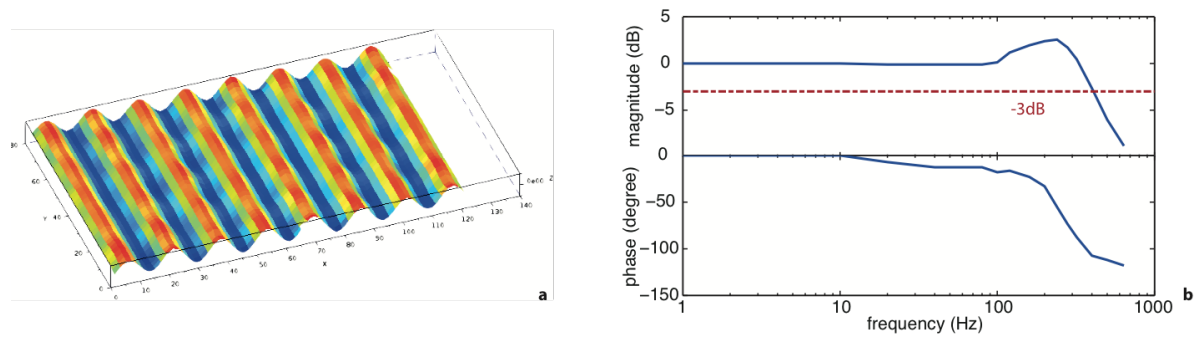

$4.2 \quad \mathrm{U}$

Itrason

ic

Vibrati

ng

Plate

The

Fig. 3. a, cartography of the ultrasonic vibrating plate. b, Bode diagram of the vibration response of the plate. The dashed line at $-3 \mathrm{~dB}$ indicates the bandwidth of the plate up to $400 \mathrm{~Hz}$.

ultra-

sonic

vibrat-

ing plate implemented in the device is a glass plate $154 \times 81 \times 1.6 \mathrm{~mm}^{3}$ resonating at $60750 \mathrm{~Hz}$, where the half wavelength of the vibration mode is $8 \mathrm{~mm}$. 22 piezoceramics, $14 \times 6 \times 0.5 \mathrm{~mm}^{3}$, are mounted on the sides of the plate along a full wavelength, 20 of which are used as motors and 2 as vibration sensors. Their unglued electrode was split along the nodal line and both halves connect- 
ed to 2 complementary outputs of the voltage inverter. This setup avoids the needing of electrical access to the glued electrode, while maintaining it at ground voltage in order to reduce perturbations to the capacitive sensor. The cartography of the vibration amplitude of the plate is reported in figure 3a. A closed loop control on the vibration amplitude, running at $60 \mathrm{kHz}$, is implemented on the microcontroller acquiring the value of the vibration from two piezoceramics used as sensors. The controller is a PI and its parameters were tuned with the Ziegler-Nichols method. In figure $3 \mathrm{~b}$, the bode diagram of the controlled system is reported. The system exhibits a bandwidth of $400 \mathrm{~Hz}$ at $2 \mu \mathrm{m}$. The closed loop control allows the stability of the vibration amplitude within a tolerance of $50 \mathrm{~nm}$ for a normal force applied lower than $3 \mathrm{~N}$ by the fingertip.

\subsection{Model Inversion}

In this subsection, the open loop control of the friction coefficient implemented in the device is

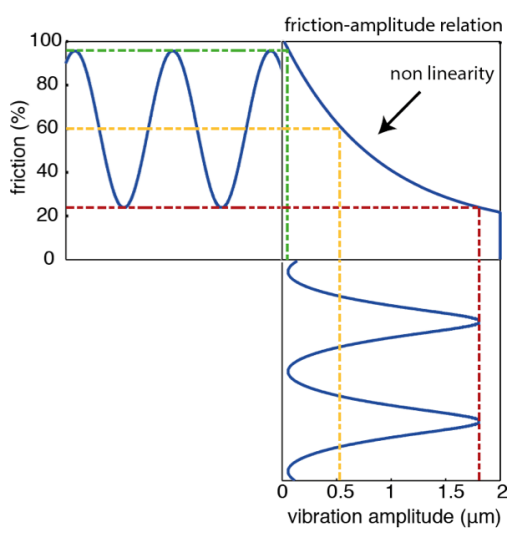

de-

scrib

ed.

$\mathrm{T}$

he

iden

tifi-

cati-

on

of

the

in-

var-

ianc

Fig. 4. Relation between reduction of friction coefficient and vibration amplitude for a given e of normal force and exploration speed. the

rela-

tive modulation of the friction coefficient allows the implementation of an inverse model on the control chain of the device. Once selected the desired percentage of friction modulation, the related vibration amplitude can be determined through an inversion of (2):

$$
w=-\frac{1}{b} \ln \frac{\Delta \mu-\Delta \mu_{1}}{1-\Delta \mu_{1}}
$$

where the constant $\Delta \mu_{1}$ is dependent on the normal force applied by the finger, figure 4 .

\subsection{Haptic Signal Representation Strategies}

In this section, two different strategies for texture reproduction are introduced.

\subsubsection{Map Representation - SHO}

In this subsection, the definition of the surface haptic object (SHO) is reported.

The classical strategy to reproduce texture and object on a flat surface through friction modulation relies on the comparison of the detected position with a map previously selected, figure $5 \mathrm{a}$. It 
is possible to introduce a concept of the SHO to describe this control strategy. We shall define as SHO a spatially located haptic activated area. The reproduction of SHO relies on the accuracy and the bandwidth of the position acquisition system to reproduce textures. This leads to the implementation of different solutions to maximize the bandwidth of the position acquisition with an optical [14] or force based [15] position detection solutions, effective, but hardly implementable on mass produced devices. A noisy or low bandwidth system cannot reproduce accurately the desired signal; e.g. a $50 \mathrm{~Hz}$ capacitive touch screen is only able to reproduce a grating up to $25 \mathrm{~Hz}$. This value corresponds in the spatial domain to a grating of $1 \mathrm{~mm}$ period for a finger velocity of 2 $\mathrm{mm} / \mathrm{s}$.

\subsubsection{Signal Synthesis - SHT}

In this subsection, the definition of the surface haptic texture (SHT) is introduced.

A different approach on the texture reproduction is implemented through a synthesis of the haptic signal based on a single period. It has been shown that the interaction with a spatially periodical texture of the fingertip leads to a periodical spatial pattern of the lateral force [16]. Through a synthesis based on the periodic force signal it is possible to avoid the limitations imposed by a low bandwidth position sensor, figure $5 \mathrm{~b}$. As SHT, we define a spatial frequency haptic pattern independent on the finger position. The reproduction rate of the identified friction period is determined by the velocity of the finger, which is updated to the new value at each cycle of acquisition. By implementing this approach, it is possible to reproduce the full bandwidth of a periodical tactile signal with capacitive touch sensor. The drawback of this approach is the error in the spatial phase of the signal, which is related to the reintegration of the velocity without considering the position of the finger at contact, and the difference between the measured and real velocity of the finger. This is not problematic for an opaque tactile stimulator, where there is not a visual clue given by a screen of the local placement of the friction interface. However, for a transparent stimulator coupled with a screen, the errors introduced for a low spatial frequency, e.g. 1 interface $/ \mathrm{cm}$, may be easily perceivable.

\subsubsection{Composed Control}

The definition of two conceptually different surface haptic signals, SHO and SHT, allows us to theoretically define a merged control. A control composed by the linear superposition of SHT and SHO allows to obtain a broad spatial reproduction bandwidth. Following [17], the spatial bandwidth separation between SHO and SHT can be attested by the single object separation of 3-4mm. A greater spatial frequency is identified as texture, whereas a smaller is identified as single objects scattered on a flat surface. A psychophysical experiment validating this limit will be proposed in the next section.
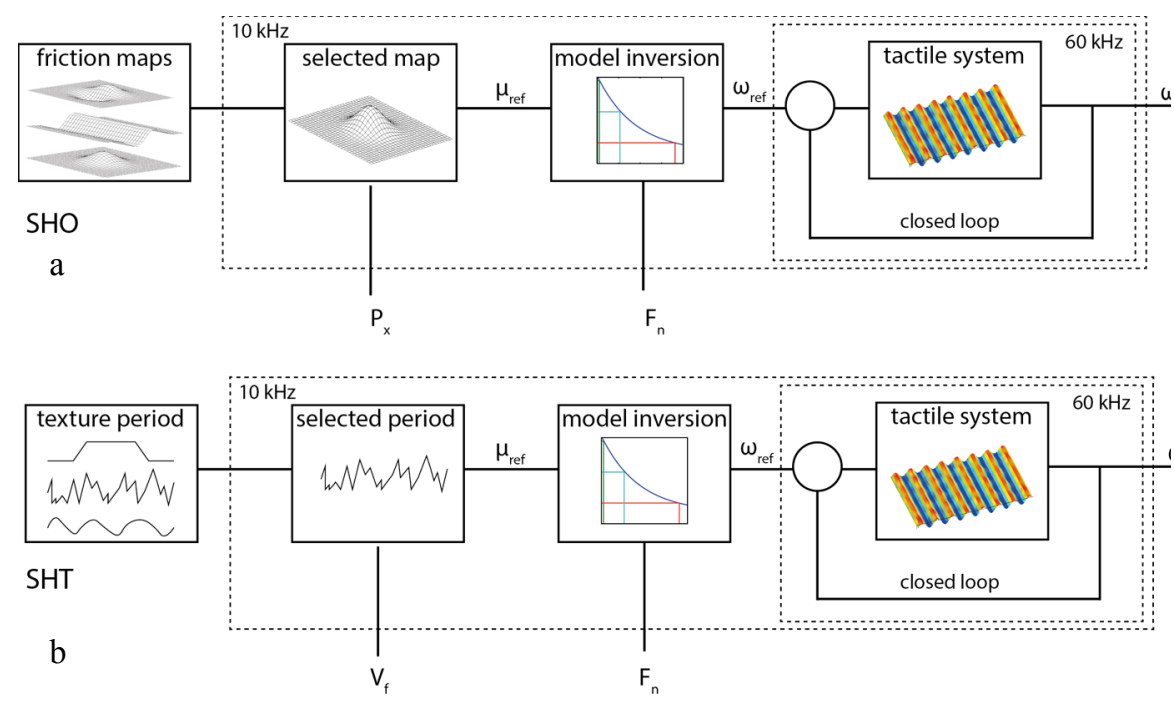

Fig. 5. a, structure of the control chain for friction coefficient modulation through map representation (SHO). b, structure of the control chain for friction coefficient modulation through period synthesis (SHT). The rate of reproduction of the selected period is dependent on the speed of exploration of the finger. 


\section{Texture Rendering Strategies Validation}

A psychophysical experiment was developed to validate the advantages and disadvantages of the rendering strategies SHO and SHT. It was asked to 6 participants to freely evaluate, with a number from one to ten, the accuracy of reproduction of three different spatialized tactile signals, which gave their informed consent to the participation to the experiment. During the exploration, a visual grating was shown on the screen of the device with the same spatial frequency as the tactile grating, figure 6a. The tactile signals were programmed to provide a step friction change at the interface between a black and white line, figure $6 \mathrm{~b}$. The participants were free to accustom themselves with the sensation provided by the device before the beginning of the experiment with a simple visual and tactile interface in the middle of the screen. The three analyzed cases were, respectively, composed by a spatial grating of 15,5 and $0.6 \mathrm{~mm}$, all of them reproduced with both

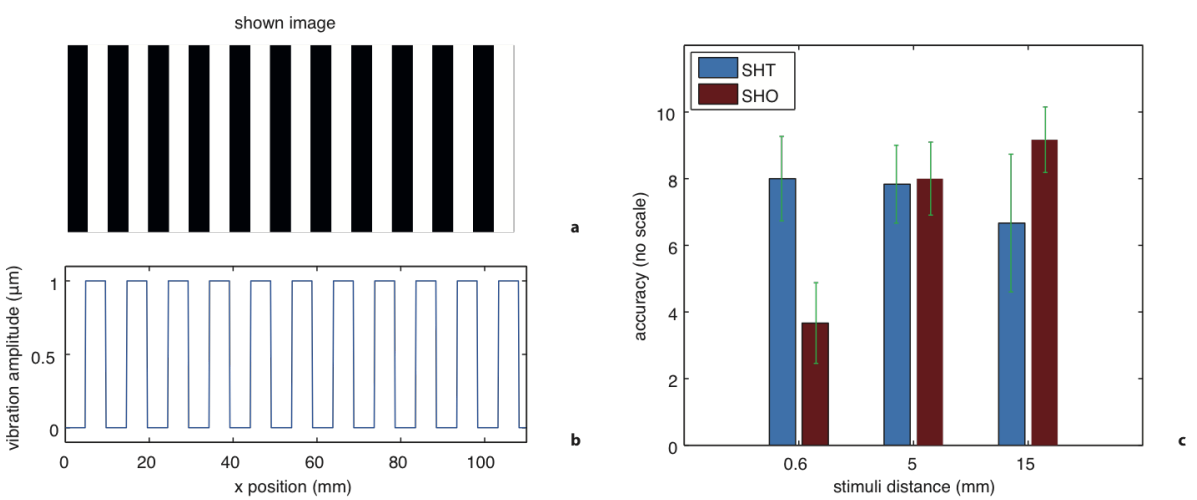

Fig. 6. a image shown on the screen, $\mathbf{b}$ envelope of the vibration amplitude in function of the $\mathrm{x}$ position of the finger, $\mathbf{c}$ given rate of reproduction accuracy for SHO and SHT. strate-

gies.

The signals were presented randomly to the users which were allowed to explore

them freely. At the end of each exploration, it was asked to the users to rate, with the number from one to ten, the accuracy of reproduction of the signals. The results are reported in figure 6c. The difference between the control strategies was tested with an unpaired t-test. For the lowest spacing, the SHT performs significantly better than SHO $(p=0.0001)$. Whereas for the higher spacing SHO performs better than SHT $(p=0.0232)$. No significant difference between the two strategies was found for the intermediate case $(\mathrm{p}=0.8)$.

\section{Discussion}

The empirical model describing the behaviour of the friction reduction is included in the control scheme of the friction force of an ultrasonic friction modulation device. This inclusion allows to overcome the limitations of a closed loop approach of the friction coefficient control in terms of bandwidth and a robust friction coefficient control up to $400 \mathrm{~Hz}$ is designed.

The approach to texture simulation on friction control devices used to be based on the comparison of the finger position with a precompiled map of friction. This approach is effective, but the bandwidth of the position sensor determines the maximum reproduction bandwidth of the device. 
In the case of capacitive touchscreen, this value is in the order of $50 \mathrm{~Hz}$, largely insufficient to reproduce real textures. By defining two different signals, the SHO, spatially located, and the SHT, spatially periodical, it is possible to overcome the limitation of the slow position acquisition through the introduced texture rendering strategy.

The advantages of the different control strategies in function of the spatial frequency of the stimulus were investigated with a psychophysical experiment. The SHT approach performs significantly better than the SHO for a large spatial frequency $(17 \mathrm{stimuli} / \mathrm{cm})$ resolving the undersampling problem of the finger position. The SHO approach is better than the SHT for a spatial frequency around $(0.7 \mathrm{stimuli} / \mathrm{cm})$ where the phase error accumulated by SHT becomes noticeable. No statistical difference was found for the intermediate case. This allows us to set a spatial frequency boundary between SHO and SHT, located around 2 stimuli/cm in accord with perception separation described in [17].

This results here shown present a fundamental difference to a related experience introduced by Meyer et al. [18]. In the cited work, the interest of the author was on the identification of the smallest spatial gap impossible to identify by the user as different from the provided noise. The aim of their experience was to highlight the passage from a spatial encoded regime, to a fully vibrational regime. The present work is totally focussed on the spatial texture perception provided by periodic signals. In this work, the participants were not able to perceive the difference between a spatial signal where the tactile stimulation was provided on the white/black interface or consistently in another spatial spatial phase of the period, accordingly with the hypothesis at the basis of the SHT definition.

The boundary between SHO and SHT placed around a spatial density of $5 \mathrm{~mm}$ is understandable by considering that the friction modulation device does not provide a localisable stimulus under the fingerpad, but provide a change of the frictional boundary condition of the whole area in contact. The inability to place the stimulus in a determined area of the finger induce the inability to distinguish the spatial phase error of the SHO signal when at least two expected stimuli are present under the fingerpad area.

The results confirm that the necessity of a high bandwidth position recording is not necessary to provide dense spatial stimuli information, provided that the reproduced spatial signal can be described as a periodic signal. This result is consistent with the [16] and do provide a direct experimental proof of the introduced concept.

The developed decomposition of the haptic signal is implementable in every friction-based technique, and can overcome low fidelity of texture reproduction currently implemented in these devices for periodical signals.

\section{$7 \quad$ Prospective}

A future work with the developed device will be to identify a joint control strategy and digital texture representation unifying the advantages of SHO and SHT. The device itself is a versatile tool that will be employed in multiple works on the interaction with haptic devices. The advantages of the synthesis-based rendering approach will be shown with a demonstrator at the conference. 


\section{Conclusion}

A device incorporating an open loop control of the friction force between the finger sliding on an ultrasonic vibrating plate is proposed. Two different texture rendering schemes are introduced, a classic one for object representation and a digital synthesis for texture representation. To conclude, the advantages of the two texture rendering techniques are analysed through a psychophysical experiment.

\section{$9 \quad$ Acknowledgement}

This work was founded by the FP7 Marie Curie Initial Training Network PROTOTOUCH, grant agreement No. 317100 and supported by IRCICA USR 3380 Univ.Lille - CNRS (www.ircica.univ-lille1.fr)

\section{References}

[1] E. Mallinckrodt, A. L, and W. Sleator Jr., "Perception by the skin of electrically induced vibrations," Science, vol. 118, pp. 277-278, 1953.

[2] E. Vezzoli, M. Amberg, F. Giraud, and B. Lemaire-Semail, "Electrovibration Modeling Analysis," in Haptics: Neuroscience, Devices, Modeling, and Applications, M. Auvray and C. Duriez, Eds. Springer Berlin Heidelberg, 2014, pp. 369-376.

[3] C. D. Shultz, M. A. Peshkin, and J. E. Colgate, "Surface haptics via electroadhesion: Expanding electrovibration with Johnsen and Rahbek," in 2015 IEEE World Haptics Conference (WHC), 2015, pp. $57-$ 62.

[4] M. Biet, F. Giraud, and B. Lemaire-Semail, "Squeeze film effect for the design of an ultrasonic tactile plate," IEEE Trans. Ultrason. Ferroelectr. Freq. Control, vol. 54, no. 12, pp. 2678-2688, Dec. 2007.

[5] E. Vezzoli, B. M. Dzidek, T. Sednaoui, F. Giraud, M. Adams, and B. Lemaire-Semail, "Role of Fingerprint Mechanics and non-Coulombic Friction in Ultrasonic Devices," WHC 2015.

[6] M. Biet, G. Casiez, F. Giraud, and B. Lemaire-Semail, "Discrimination of Virtual Square Gratings by Dynamic Touch on Friction Based Tactile Displays," in symposium on Haptic interfaces for virtual environment and teleoperator systems, 2008. haptics 2008, 2008, pp. 41-48.

[7] G. Robles-De-La-Torre and V. Hayward, "Force can overcome object geometry in the perception of shape through active touch," Nature, vol. 412, no. 6845, pp. 445-448, Jul. 2001.

[8] T. Watanabe and S. Fukui, "A method for controlling tactile sensation of surface roughness using ultrasonic vibration," in , 1995 IEEE International Conference on Robotics and Automation, 1995. Proceedings, 1995, vol. 1, pp. 1134-1139 vol.1.

[9] T. Sednaoui, E. Vezzoli, B. M. Dzidek, B. Lemaire-Semail, C. Chiappaz, and M. Adams, "Experimental evaluation of friction reduction in ultrasonic devices," in World Haptics Conference (WHC), 2015.

[10] B. M. Dzidek, M. Adams, Z. Zhang, S. Johnson, S. Bochereau, and V. Hayward, "Role of Occlusion in Non-Coulombic Slip of the Finger Pad," in Haptics: Neuroscience, Devices, Modeling, and Applications, M. Auvray and C. Duriez, Eds. Springer Berlin Heidelberg, 2014, pp. 109-116.

[11] W. Ben Messaoud, B. Lemaire-Semail, M.-A. Bueno, M. Amberg, and F. Giraud, "Closed-Loop Control for Squeeze Film Effect in Tactile Stimulator," ACTUATOR 2014, BREMEN. 
[12] M. Wiertlewski and V. Hayward, "Mechanical behavior of the fingertip in the range of frequencies and displacements relevant to touch," J. Biomech., vol. 45, no. 11, pp. 1869-1874, Jul. 2012.

[13] E. Vezzoli, W. Ben Messaoud, M. Amberg, B. Lemaire-Semail, F. Giraud, and M.-A. Bueno, "Physical and perceptual independence of ultrasonic vibration and electrovibration for friction modulation," IEEE - Transaction on Haptics, Jun-2015.

[14] M. Wiertlewski, D. Leonardis, D. J. Meyer, M. A. Peshkin, and J. E. Colgate, "A High-Fidelity Surface-Haptic Device for Texture Rendering on Bare Finger."

[15] M. Amberg, F. Giraud, B. Semail, P. Olivo, G. Casiez, and N. Roussel, "STIMTAC: A Tactile Input Device with Programmable Friction," in Proceedings of the 24th Annual ACM Symposium Adjunct on User Interface Software and Technology, New York, NY, USA, 2011, pp. 7-8.

[16] M. Wiertlewski, J. Lozada, and V. Hayward, "The Spatial Spectrum of Tangential Skin Displacement Can Encode Tactual Texture," IEEE Trans. Robot., vol. 27, no. 3, pp. 461-472, Jun. 2011.

[17] R. L. Klatzky and S. J. Lederman, "Touch," in Handbook of Psychology, John Wiley \& Sons, Inc., 2003.

[18] D. J. Meyer, M. A. Peshkin, and J. E. Colgate, "Modeling and Synthesis of Tactile Texture with Spatial Spectrograms for Display on Variable Friction Surfaces," IEEE - World Haptics Conf. 2015. 